\title{
Development of a Potential Therapeutic Small Volume Resuscitative Fluid against Hemorrhagic Shock in Rat Model
}

\author{
Mohamed Shirhan Bin Mohamed Atan ${ }^{1}$ and Shabbir M Moochhala ${ }^{2,3 *}$ \\ ${ }^{1}$ School of Applied Sciences, Temasek Polytechnic, Singapore \\ ${ }^{2}$ Department of Pharmacology National University Singapore, Singapore \\ ${ }^{3}$ Department of Surgery, National University Hospital, Singapore \\ *Corresponding author: Shabbir Moochhala, Department of Surgery, Yong Loo Lin School of Medicine, NUHS Tower Block, 1E, \\ Kent Ridge Wing, Singapore 119228, Singapore
}

\section{ARTICLE INFO}

Received: 蔧 June 10, 2020

Published: 幽 June 23, 2020

Citation: Mohamed Shirhan Bin Mohamed Atan, Shabbir M Moochhala. Development of a Potential Therapeutic Small Volume Resuscitative Fluid against Hemorrhagic Shock in Rat Model. Biomed J Sci \& Tech Res 28(3)-2020. BJSTR. MS.ID.004653.

Keywords: Aminoguanidine; Hypertonic saline; Haemorrhagic shock; Nitric oxide; Rat
ABSTRACT

Objective: Excessive production of nitric oxide (NO) as result of inducible nitric oxide synthase (iNOS) induction has been implicated in the pathophysiology of hemorrhagic shock. Our aim was to study the effect of iNOS inhibitor, aminoguanidine (AG) when combined with a resuscitation fluid, hypertonic saline (7.5\%HTS), on biochemical, hemodynamic and neurological functions in rodents subjected to hemorrhagic shock.

Materials and methods: Hemorrhagic shock was achieved via a constant pressure method. Neurological and behavioural tests were performed 24, 48 and 72-post hemorrhagic shock period. Plasma collected from hemorrhagic shocked rats was used to measure nitrate/nitrite, $\mathrm{GOT}, \mathrm{pO}_{2}$ and $\mathrm{pH}$ levels.

Results: AG+HTS-treated rats had significantly improved biochemical, blood gases and hemodynamic outcomes when compared to untreated rats following hemorrhagic shock. A marked increase in the neurological performance was observed in AG+HTStreated rats over the three-day period when compared with all treatment groups. Conclusions: AG+HTS-treated rats were able to improve physiological and neurological outcome. It is therefore suggested that AG+HTS may be beneficial as a potentially useful small volume resuscitative fluid in improving hemodynamic and neurological deficit in hemorrhagic shock.

\section{Introduction}

Hemorrhagic Shock (HS) is a major cause of death following trauma and it is characterized by severe hypotension, loss of vascular reactivity and systemic tissue damage [1,2]. It is estimated that approximately $20 \%$ of these deaths are preventable if bleeding is quickly controlled and given proper resuscitation $[1,3]$. Hypertonic fluids offer a major advantage over isotonic fluids as a small volume is required for resuscitation [4]. Numerous studies have shown that intravenous administration of hypertonic saline is safe in dehydrated animal models of hemorrhagic shock. Also, it has now been recognized that small-volume hypertonic resuscitation is an effective means for restoration of cardiovascular function and hemodynamic parameters after hemorrhage [5-9]. Infusion of hypertonic saline (HTS) solutions lead to an instantaneous mobilization of fluids from the intracellular to extracellular compartments by the osmotic gradient produced by HTS, which caused rapid and significant plasma expansion, an increase in oxygen delivery, and elevated cardiac output and increased oxygen extraction [10].

Another strategy is to use resuscitation fluids that can both replace intravascular volume and protect against cellular injury caused by high concentrations of nitric oxide (NO) generated during shock. Under normal conditions, a small amount of NO 
is produced in endothelial cells by the constitutively expressed NO synthase (NOS) enzyme that is important in maintaining the integrity of blood flow through the microcirculation by regulating resistance vessel diameter, blood rheology, interaction between cellular blood elements and the vascular wall, and blood volume [11]. However, during hemorrhagic shock, there is an increase in NO due to cytokines such as IL-1, IL-6, TNF- $\alpha$, INF- $\gamma$, IL-1 $\beta$ and endotoxin (lipopolysaccharide), which increase inducible NOS (iNOS) expression. Excessive NO synthesis contributes to excessive vasodilation, loss of systemic vascular resistance, and vascular leakage [12]. High doses of NO can also act as a negative iontrope on the myocardium [13]. Pathologic levels of NO can also induce hepatocyte damage and increase gut epithelial, promoting translocation of endotoxin from the gut to circulation, contributing to high mortality rates [14]. NO synthesis is also a key event in the subsequent activation of inflammatory cascades that contributes to organ injury and early multi-organ failure [15].

Neurotoxicity of NO overproduction in the brain has also been shown by several studies [16-18]. NO may play an important role in secondary neuronal damage after ischemia [19]. Excess production of NO by inappropriate induction of NOS protein in the brain may be involved in glutamate neurotoxicity and is responsible for neuronal death [20]. The neuroprotective properties of NOS inhibitors arise from their ability to inhibit the excess release of NO after brain injury. Neuroprotective properties of NOS inhibitors have been demonstrated in several experimental animal models of ischemia $[19,21,22]$. Given the role of NO as a potent vasodilator immunomodulator, and cytotoxin, our lab has performed studies to investigate the effects of NO blockade using the iNOS selective inhibitor aminoguanidine (AG) in rat hemorrhagic shock models. We found that the injection of AG after hemorrhagic shock attenuated hypotension, mesenteric blood flow alterations, and improved survival rates. Further, NO lessened microscopic markers of organ damage and lowered the levels of GOT (markers of heart, renal, and liver impairment) and creatinine (myocardial cell injury and renal failure) [23].

We compared the effectiveness of HTS combined with AG in preserving hemodynamic and neurological functions in a rat hemorrhagic shock model. Lactated Ringer's solution was also administered during shock as a conventional resuscitative fluid treatment. The efficacy of these treatments on hemodynamic, biochemical parameters neurological performance were examined.

\section{Materials and Methods}

\section{Chemicals and Reagents}

AG was purchased from Sigma-Aldrich (St Louis, M0). The drug was dissolved in $0.9 \% \mathrm{NaCl}$ at concentration of $50 \mathrm{mg} / \mathrm{ml}$. HTS was made of 7.5\% sodium chloride. Lactate Ringers Solution from B. Braun (B. Braun, Melsungen AG, Germany). Clinical Research Centre (CRC) Cocktail consists of 1 part hypnorm (Jansen Pharmaceutica,
Beerse, Belgium) which contained $0.315 \mathrm{mg} / \mathrm{ml}$ fentanyl and $10 \mathrm{mg} /$ $\mathrm{ml}$ fluanisone, 1 part midazolam (Roche, Basel, Switerland) which contained $5 \mathrm{mg} / \mathrm{ml}$ dormicum, 2 parts water for injection. Nitrate/ Nitrite assay kit was purchased from Cayman Chemical (Ann Arbor, MI). Glutamic oxalacetic transaminase (GOT) was measured using Sigma Diagnostic kit, and the activities were expressed in SigmaFrankel (SF) units. One SF unit of GOT will form $4.82 \times 10^{-4} \mu \mathrm{mol}$ glutamate per minute at $\mathrm{pH} 7.5$ and $25{ }^{\circ} \mathrm{C}$.

\section{Animal Preparation}

The experiment described in this article was performed in adherence to the guidelines of Council for International Organization of Medical Sciences (CIOMS) ethical code for animal experimentation. Male Sprague-Dawley rats weighting 250-300 g were used for the study. The animals were acclimatized for at least a week before the experiment and had free access to standard laboratory chow and water. Each study was carried out using five rats per group. The animals were deprived of food for $24 \mathrm{~h}$ before the experiment but allowed free access to water. They were anesthetized by intraperitoneal (i.p) injection using CRC cocktail (3 $\mathrm{ml} / \mathrm{kg}$ ) and were maintained under anesthesia for the duration of the experiment. The total amount of anesthesia given was recorded. The body temperature was monitored by rectal thermometer and maintained at $36-37^{\circ} \mathrm{C}$ by a homeothermic blanket control unit (Harvard Apparatus, Oldham, England).

Animals were divided into 6 groups:

a. Sham operation group: animals underwent same surgical procedure but with no hemorrhagic shock

b. lactate Ringer solution group ( 3 times of blood lost) at $60 \mathrm{~min}$ after hemorrhagic shock

c. AG group: animals were injected with $A G(50 \mathrm{mg} / \mathrm{ml}, 2 \mathrm{ml} /$ $\mathrm{kg}$ ) at $60 \mathrm{~min}$ after hemorrhagic shock

d. HTS group: animals were injected with $\mathrm{HTS}(4 \mathrm{ml} / \mathrm{kg})$ at 60 min after hemorrhagic shock

e. AG+HTS group: animals were injected with AG $(50 \mathrm{mg} / \mathrm{ml}$, $2 \mathrm{ml} / \mathrm{kg})+\mathrm{HTS}(4 \mathrm{ml} / \mathrm{kg})$ at $60 \mathrm{~min}$ after hemorrhagic shock and

f. Untreated group whereby the animals undergoes hemorrhagic shock but did not receive any treatment.

\section{Animal Preparation for Anaesthetized Rats}

For the anesthetized rat hemorrhagic shock experiment, the right carotid artery and left jugular vein were exposed by blunt dissection between associated muscles. The adjacent vagus nerve was carefully dissected away from the artery. A heparinized 24G X $1.90 \mathrm{~cm}$ over-the-needle catheter (Terumo Corporation, Tokyo, Japan) was inserted into the carotid artery and jugular vein, and a three-way stopcock was attached in-line for monitoring the mean arterial blood pressure (MABP), withdrawal of blood, and administration of drugs. 


\section{Hemorrhagic Shock in Anesthetized Rats}

After a stabilizing period of $15 \mathrm{~min}$, hemorrhagic shock was induced by withdrawing blood in 2 min period until the MABP decreased to $40 \mathrm{mmHg}$, causing a hypotension. The pressure was maintained at this level for $60 \mathrm{~min}$ by withdrawing or reinfusing of shed blood as required. The total amount of blood withdrawn was then recorded.

\section{Mean Arterial Blood Pressure}

The MABP was monitored using a blood pressure (BP) transducer (MLT0380, AD Instruments, Australia) and recorded by computerized Power Lab Data Acquisition System (AD Instruments, Australia).

\section{Blood Sample Analysis}

Arterial blood samples were collected before shock, $60 \mathrm{~min}$ during shock, and 180 min after resuscitation. The blood collected was accounted in the total hemorrhage volume. The withdrawn blood was replaced by normal saline and shed blood for shamoperated rats and shocked rats, respectively. $\mathrm{pO}_{2}, \mathrm{pH}$, glucose, lactate, creatinine was measured by i-STAT Portable Clinical Analyzer (Abbott Laboratories Inc. New Jersey, USA) immediately after collection. Plasma GOT and nitrate/nitrite were measured with GOT Sigma Diagnostic kit and Nitrate/Nitrite assay kit, respectively.

\section{Rotameric Test}

A rotameric device (Columbus Instruments Rotamex 4/8 system, Ohio, USA) was used to examine the coordination ability of the animal while being placed on a rotating rod. The rotating speed of the rod was set between 5rpm (start speed) and $30 \mathrm{rpm}$ (end speed) for a period of 240 seconds. An internal micro-controller was used to detect the time at which the subject fell from the rod. The average reading (in seconds) of 3 successive trials was taken from each animal.

\section{Fore Limb Grip-Strength Test}

Forelimb grip strength was determined using a grip strength meter (Columbus Instruments, Ohio, USA). The animals were placed on the electronic digital force gauge that measures the peak force exerted by the action of the animal. While being drawn along a straight line leading away from the sensor, the animal released at some point and the maximum force attained was stored on the display. The highest reading (in Newtons) of three successive trials was taken for each animal.

\section{Neurological Severity Score (NSS)}

Animals were habituated to the test room for at least 60 minutes prior to testing in their home cages. NSS and was conducted for a 3-day monitoring period after hemorrhagic shock. The neurological status of the rats was assessed using NSS, a well-established scoring system [24] to determine the neurological performance of the rats after hemorrhagic shock. The NSS consist of tests that assess the animal's performance in mobility, reflexes, behavior and function tests that include beam walking and beam balancing, which assess fine and gross vestibular motor function and coordination. A score of 0 indicates no neurological deficit and a score of 25 indicates the most severe impairment.

\section{Locomotor Activity}

The locomotor activity of the animal was recorded by using a device that consists of a rectangular perspex with sensors along the length of the container. The rat was placed at one specific corner of the container and acclimatized for a 2-min period. Any locomotor activity made by the animal was then picked up by the sensor and was recorded for $5 \mathrm{~min}$. Total and ambulatory locomotor activities were recorded separately using two counters. Total locomotor activity refers to any movement made by the rat that is picked up by the sensors. This can include repeated activation of a single sensor. Ambulatory locomotor activity refers to the movement made by the rat as it moves between distinct sensors excluding repeated activation of a single sensor. By subtraction of the measure for ambulatory locomotor activity from the measure of total locomotor activity, a score of the grooming and rearing behavioral patterns of the animal can be calculated, given that no convulsive activity was observed.

\section{Statistical analysis}

The results are expressed as mean \pm standard deviations. Statistical analysis was performed using the Statistical Package for Social Science for Windows (SPSSWIN). One-way analysis of variance using Duncan's multiple range test were employed to compare the differences between groups. Probability values less than 0.05 were considered significant.

\section{Results}

\section{Mean arterial blood pressure (Figure 1)}

Sham-operated animals maintained MABP at around $112.1 \pm$ $4.8 \mathrm{mmHg}$ throughout the experimental period. There was a MABP recording of $47.96 \pm 5.6 \mathrm{mmHg}$ and $62.33 \pm 2.2 \mathrm{mmHg}$ after the hemorrhagic shock period in untreated and lactate rats respectively. In rats treated with $\mathrm{AG}+\mathrm{HTS}$, the MABP was $75.7 \pm 3.8 \mathrm{mmHg}, 70.8$ $\pm 3.6 \mathrm{mmHg}$ for AG rats and $61.5 \pm 3.3 \mathrm{mmHg}$ for HTS rats. Only $A G \pm H T S$ treatment group showed a significant improvement in MABP levels compared with untreated and lactate treated rats. 


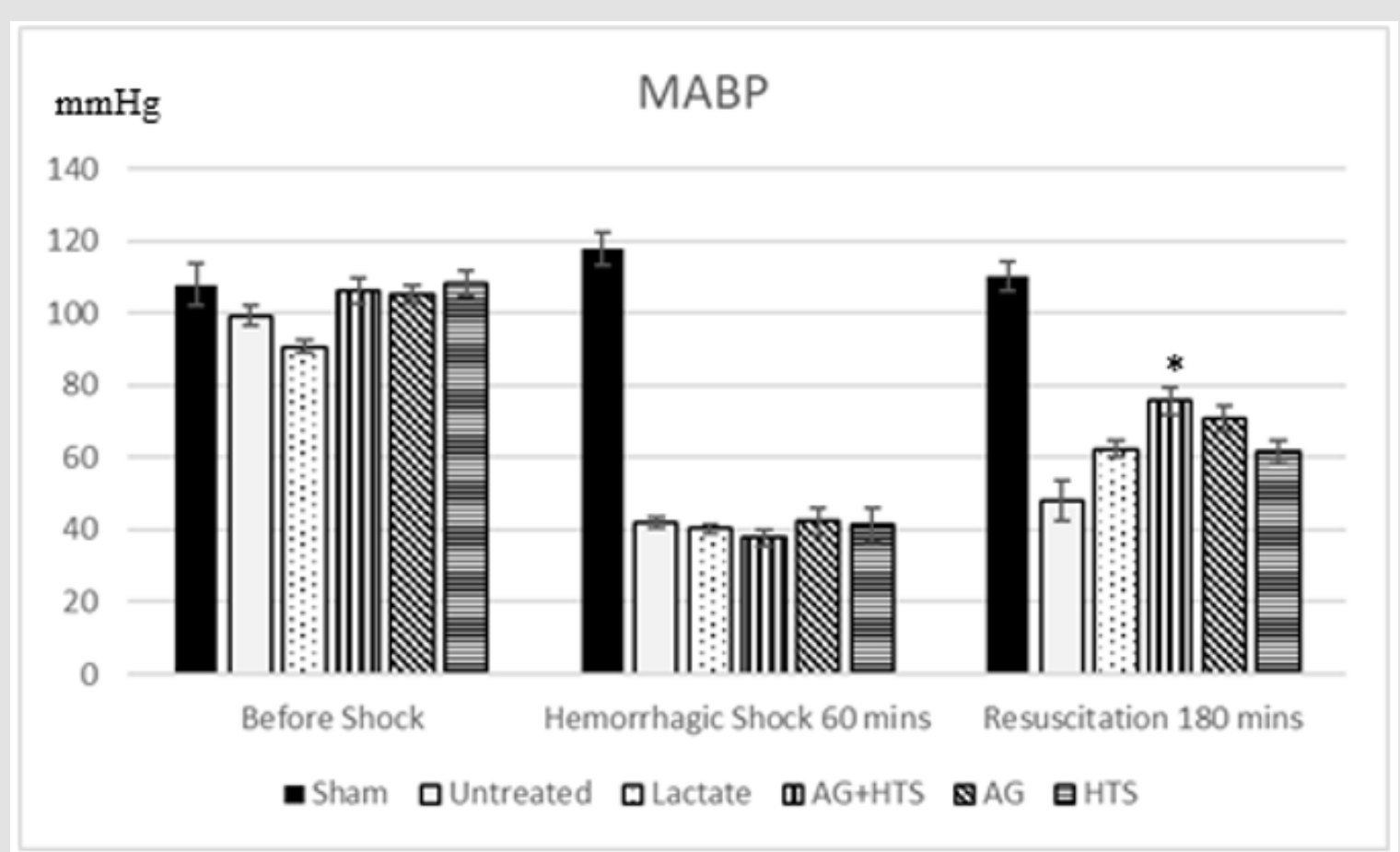

Figure 1: MABP of rats recorded before, during and after hemorrhagic shock. AG+HTS showed a significant improvement in MABP levels compared with untreated and lactate treated rats. ( ${ }^{*} \mathrm{p}<0.05$ vs untreated and treated groups).

\section{Nitrate/Nitrite Determination (Figure 2)}

The plasma nitrate/nitrite level in sham-operated rats was $0.77 \pm 0.06 \mu \mathrm{M}$. There was no significant difference $(\mathrm{P}>0.05)$ between sham-operated and non-operated rats (data not shown).
The plasma nitrate/nitrite production was significantly increased in untreated hemorrhagic shocked rats $(1.35 \pm 0.11 \mu \mathrm{M})$. AG+HTS treatment resulted in a significantly lower production of nitrate/ nitrite when compared to untreated and HTS rats but not with lactate rats.

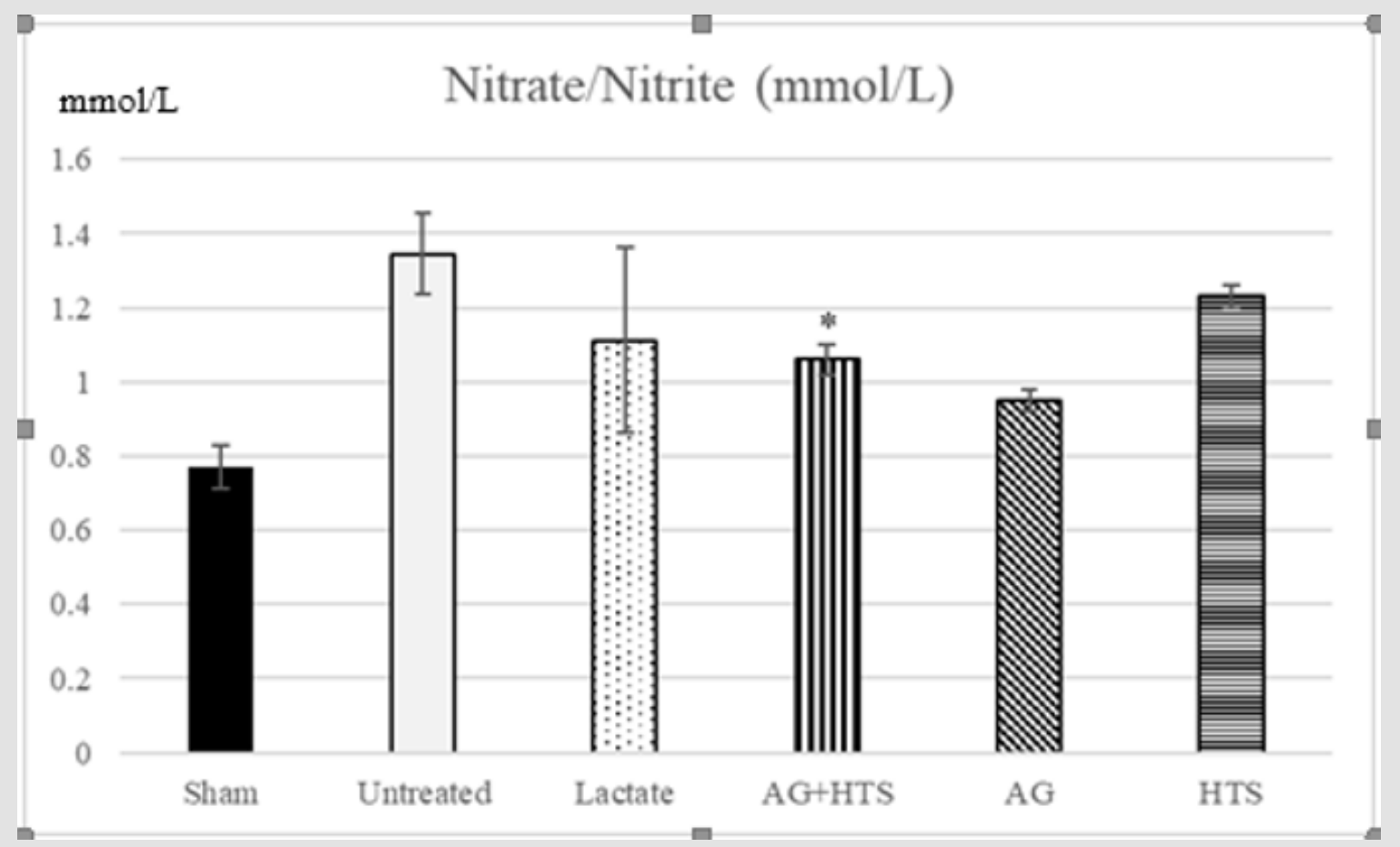

Figure 2: The plasma nitrate/nitrite level showed AG+HTS treatments resulted in a significantly lower production of nitrate/ nitrite when compared to untreated and HTS rats but not with lactate rats. ( ${ }^{*} p<0.05$ vs untreated and HTS-treated groups). 


\section{Glutamic Oxalacetic Transaminase (Figure 3)}

GOT activity was found to be $99.6 \pm 2.5 \mathrm{U} / \mathrm{ml}$ in sham-operated rat plasma. Untreated and lactate hemorrhagic shocked rats had a significantly higher GOT level $(171.1 \pm 12.1$ and $148.2 \pm 8.9 \mathrm{U} /$ $\mathrm{ml}$ ) respectively. The other treatment groups showed significant decrease in GOT levels when compared with untreated rats. A significant decrease in GOT was found only in hemorrhagic shocked rats receiving AG \pm HTS $(104.2 \pm 4.3 \mathrm{U} / \mathrm{ml})$ when compared with untreated and treated rats.

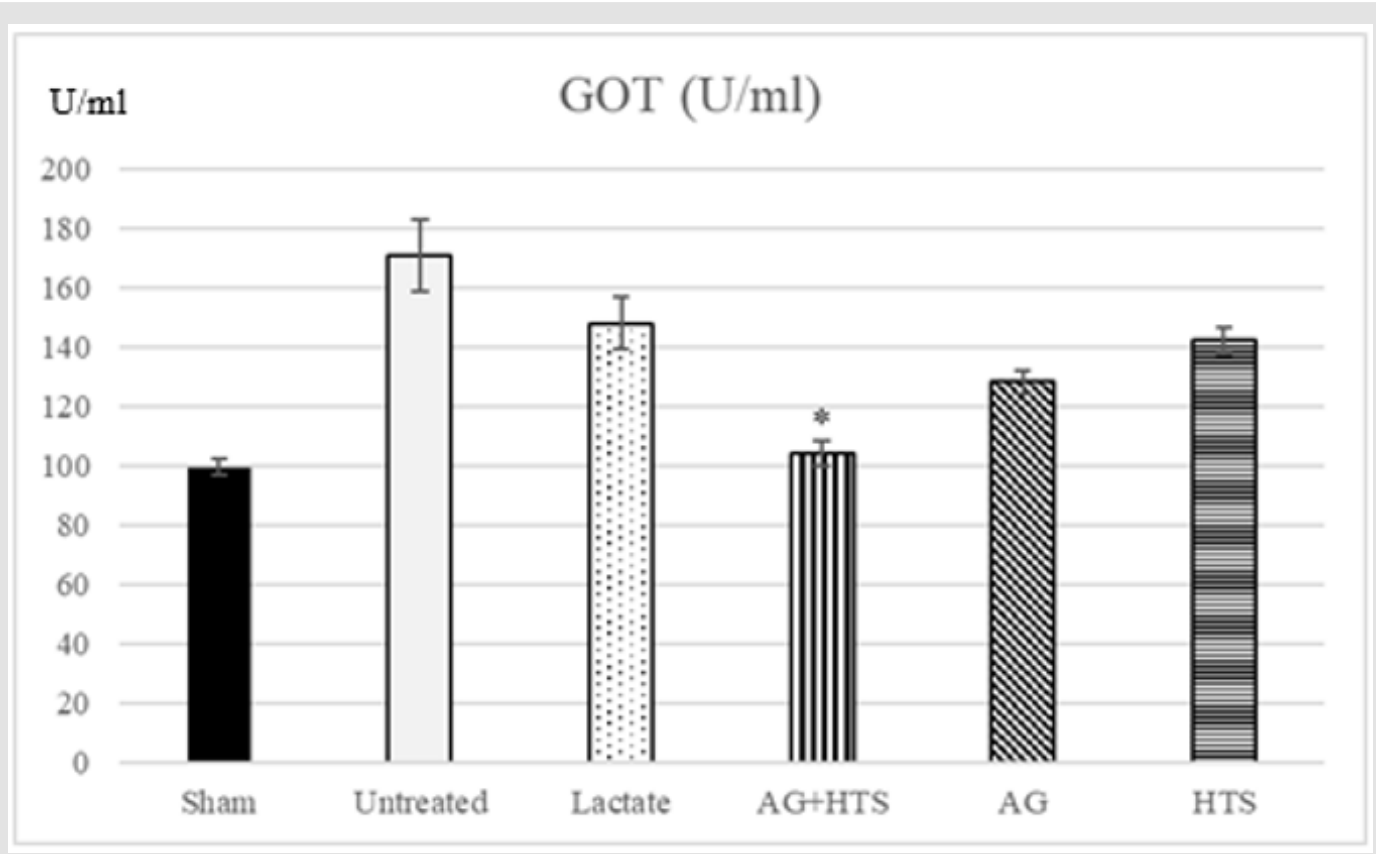

Figure 3: The plasma GOT activity showed a significant decrease in GOT was found only in hemorrhagic shocked rats receiving AG \pm HTS when compared with untreated and treated rats. ( ${ }^{*} p<0.05$ vs untreated and HTS-treated groups).

\section{pH \& p0, analysis (Figures 4 \& 5)}

$\mathrm{pH}$ and $\mathrm{pO}_{2}$, act as indicators of lung function. Untreated rats showed possible impaired lung function with and high $\mathrm{pO}_{2}(153.3 \pm$
$13.4 \mathrm{mmHg})$ and $\mathrm{pH}(7.08 \pm 0.06)$ levels compared to sham-operated rats. Although $\mathrm{AG}+\mathrm{HTS}$-treated rats showed a slightly lowered $\mathrm{pO}_{2}$, and $\mathrm{pH}$, no significant difference ( $\mathrm{p}>0.05$ ) was seen with the rest of the treated groups.

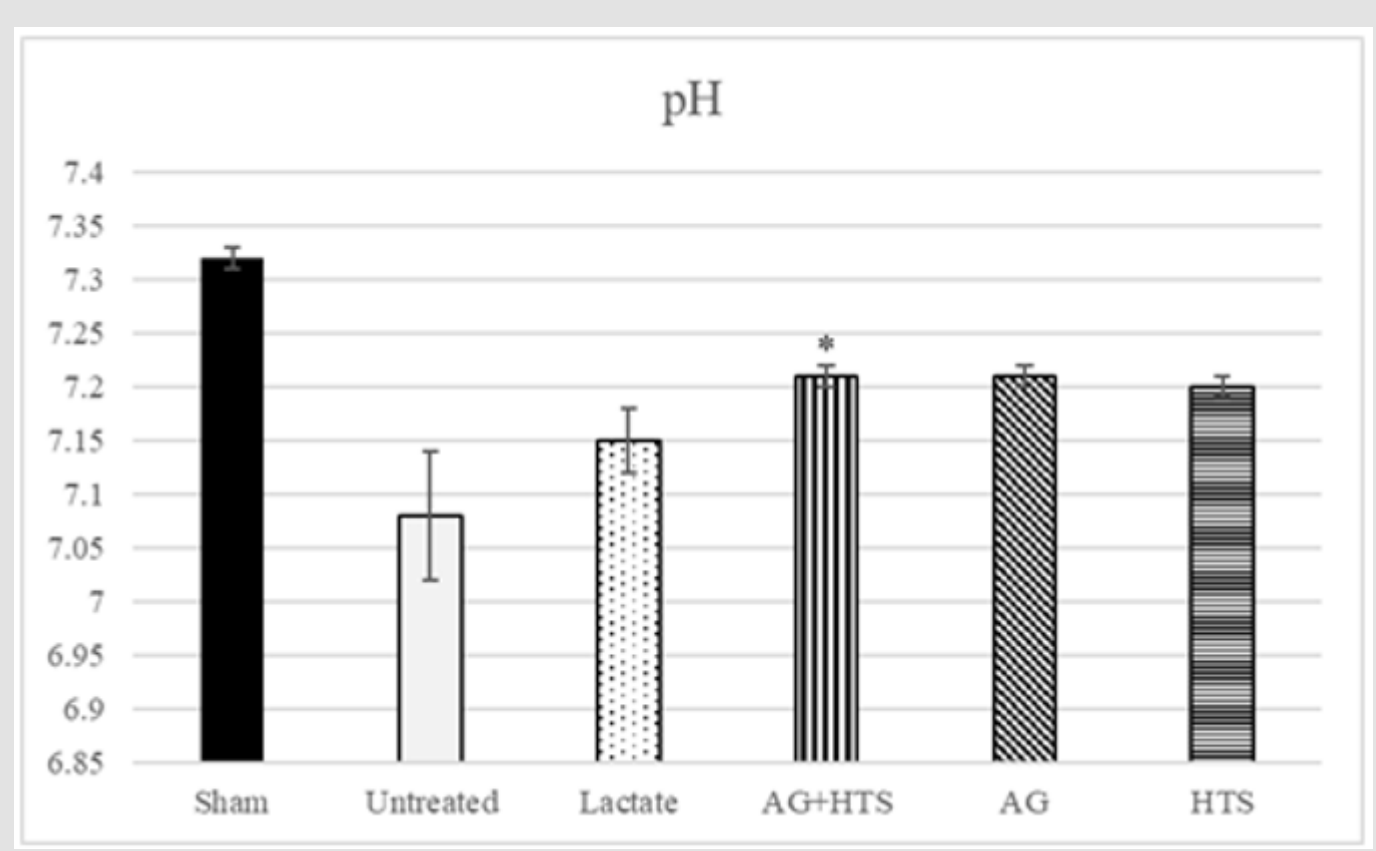

Figure 4: Untreated rats showed a low $\mathrm{pH}(7.08 \pm 0.06)$ level compared to sham-operated rats. Although AG+HTS-treated rats showed a slightly lowered $\mathrm{pH}$, no significant difference $(\mathrm{p}>0.05)$ was seen with the rest of the treated groups. 


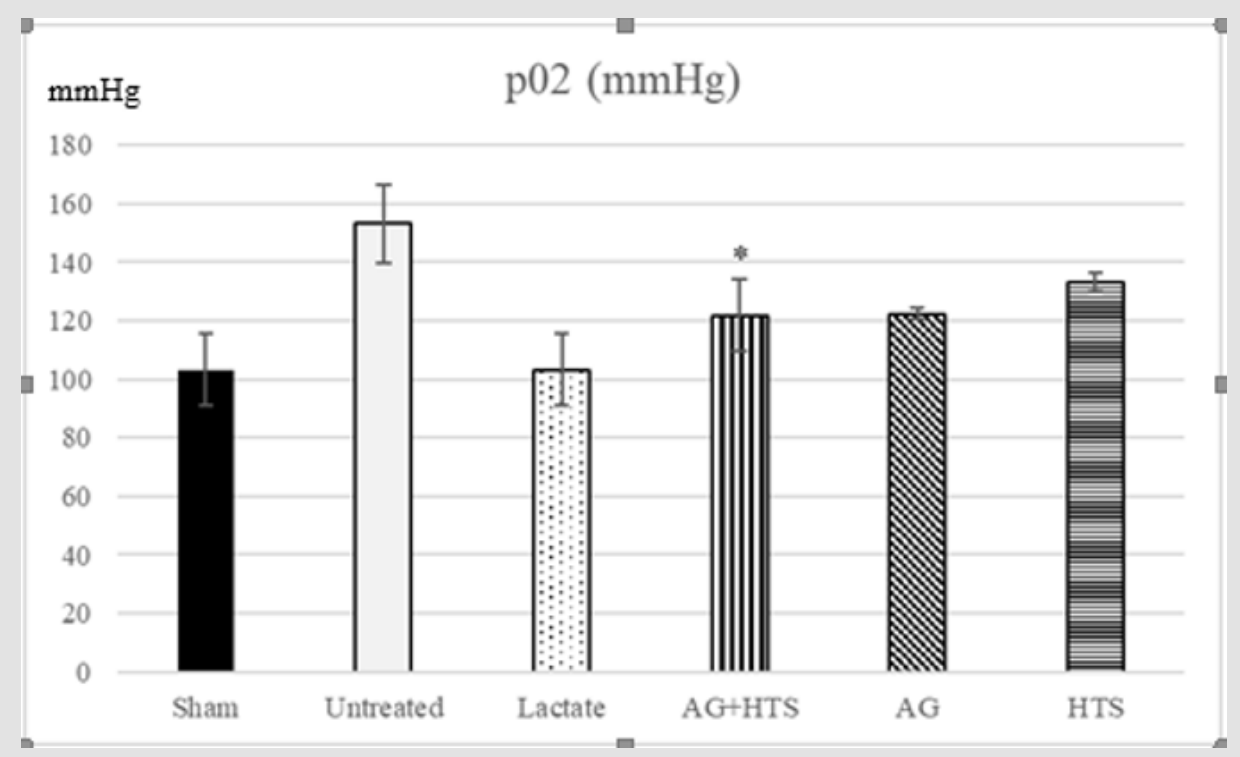

Figure 5: Untreated rats showed a high $\mathrm{pO}_{2}$ level compared to sham-operated rats. Although AG+HTS-treated rats showed a slightly lowered $\mathrm{pO}_{2}$, no significant difference $(\mathrm{p}>0.05)$ was seen with the rest of the treated groups.

\section{Neurological tests}

Animals that survived for more than 24 hours were subjected to a series of motor coordination performance and neurological assessment tests. All untreated rats were used as the active comparator.

\section{Tests}

Performance Tests (Figures 6 \& 7): Rotameric performance served as a measurement of coordination and impairment of locomotor agility by assessing the ability of a shocked animal to maintain its balance on a rotating rod. Grip strength meters were employed in assessing neuromuscular function by sensing the peak amount of force an animal applies in grasping specially designed pull-bar assemblies. Sham-operated rats recorded a mean rotameric performance of 233-238 s for the 3-day monitoring period. AG+HTS-treated rats (215.7 to $222.5 \mathrm{~s}$ ) recorded an overall significant improvement in rotameric performance for the 3-days when compared to all treatment groups. Untreated rats (170.1 to $170.6 \mathrm{~s}$ ) showed the poorest rotameric performance (Figure 6). Sham-operated rats recorded a mean grip strength score of 8.6-9.1 $\mathrm{N}$ for the 3-day monitoring period (Figure 8). AG+HTS-treated rats showed an overall significant improvement in grip strength scores of $8.6,8.6$, and $8.9 \mathrm{~N}$ at 24,48 , and 72 hours time points, respectively over all treatment rats. Untreated rats (average over the 3-day period of $5.4 \mathrm{~N}$ ) showed the poorest rotameric performance (Figure 7).

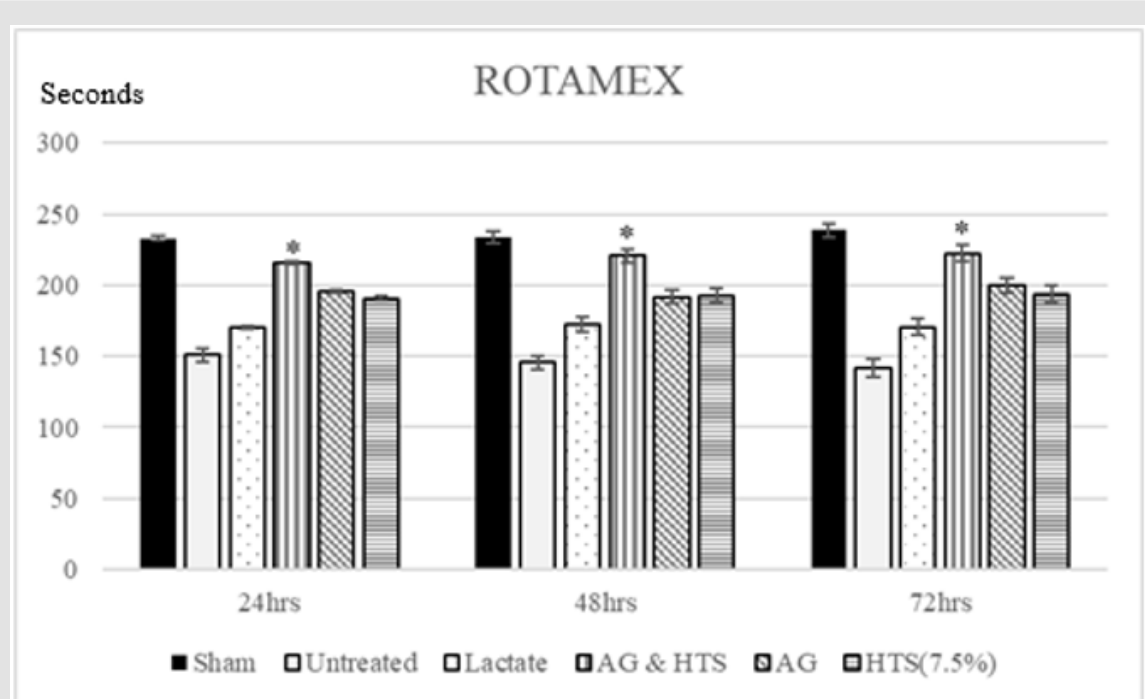

Figure 6: Rotameric performance served as a measurement of coordination and impairment of locomotor agility by assessing the ability of a shocked animal to maintain its balance on a rotating rod. AG+HTS-treated rats recorded an overall significant improvement in rotameric performance for the 3 -days when compared to untreated and all treatment groups. $\left({ }^{*} \mathrm{p}<0.05\right.$ vs untreated and treated groups). 


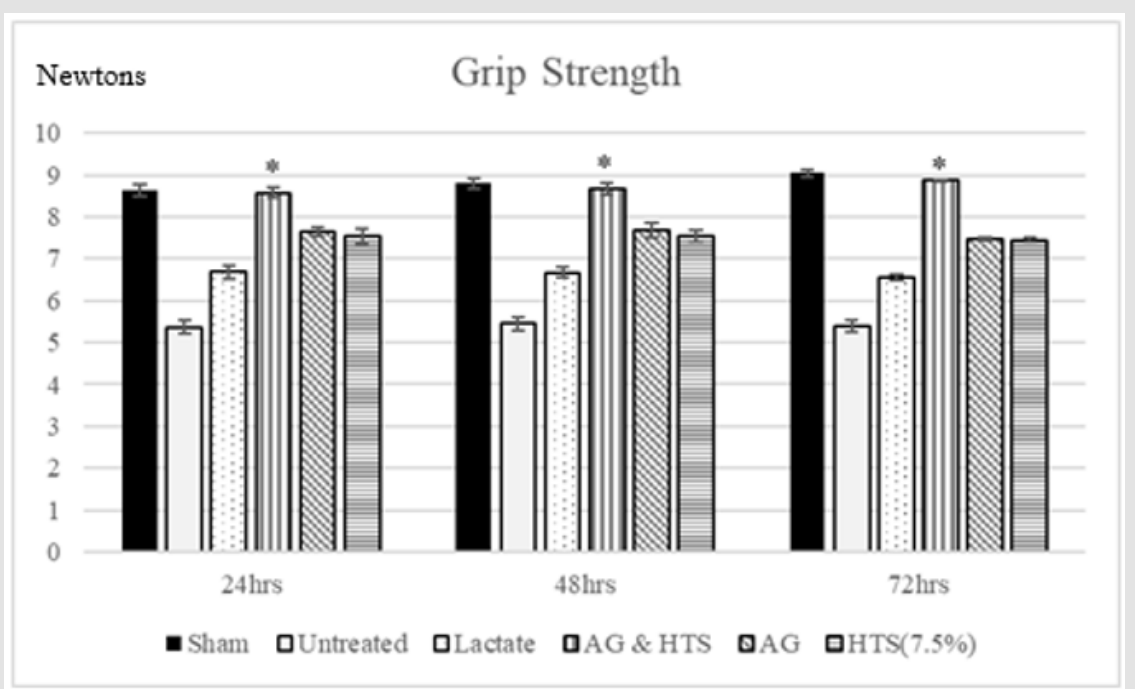

Figure 7: Grip strength meters were employed in assessing neuromuscular function by sensing the peak amount of force an animal applies in grasping specially designed pull-bar assemblies. AG + HTS-treated rats showed an overall significant improvement in grip strength scores at 24,48 , and 72 hours' time points over untreated and all treatment rats. $\left({ }^{*} \mathrm{p}<0.05 \mathrm{vs}\right.$ untreated and treated groups).

Behavioral Tests (Figure 8): Sham-operated rats recorded an NSS score of $0.8-1.2$ for the 3-day monitoring period. Neurological severity scores of AG+HTS treated rats were overall significantly lower at 24, 48, and 72 hours' time points $(3.6,4.3$, and 3.5, respectively) when compared to all treatment rats.

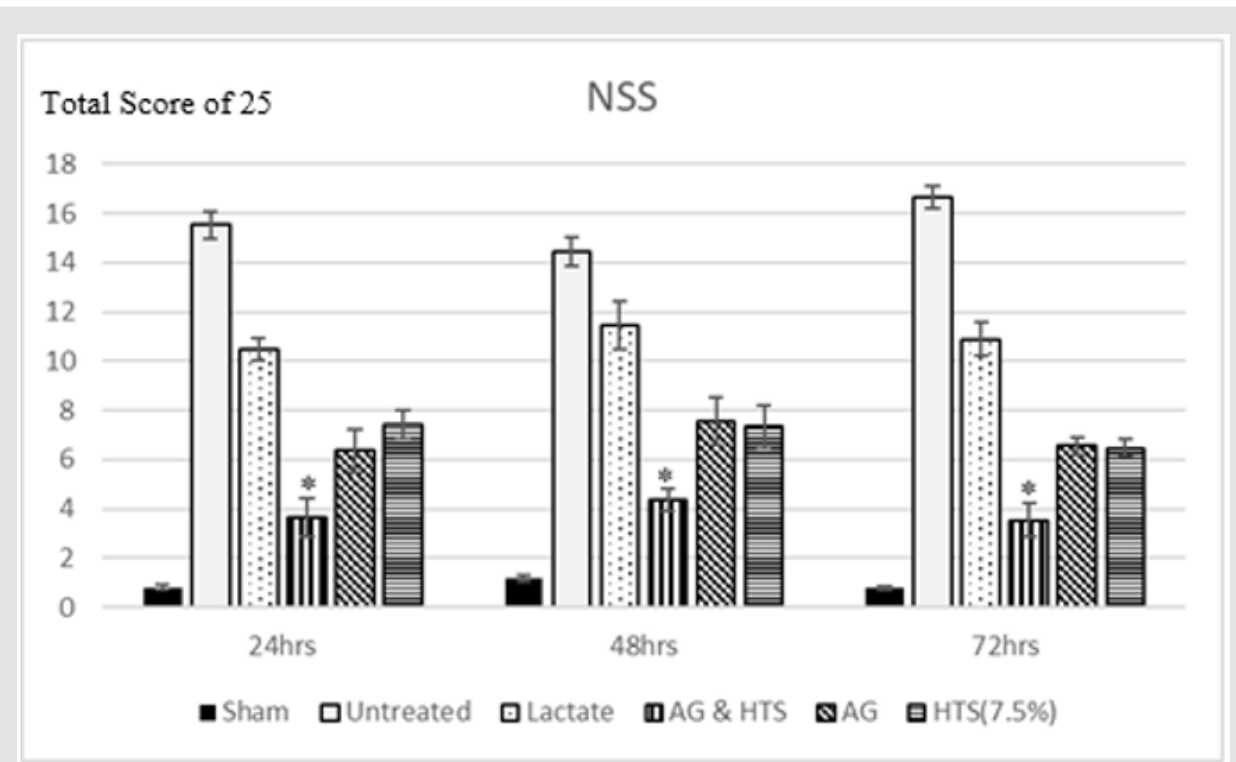

Figure 8: Neurological severity scores of AG+HTS treated rats were overall significantly lower at 24, 48, and 72 hours' time points when compared to untreated and all treatment rats. ( ${ }^{*} \mathrm{p}<0.05$ vs untreated and treated groups).

Locomotor Activity (Figures 9 \& 10): A significant increase in total (Figure 9) and ambulatory activity (Figure 10) was observed in the AG+HTS group when compared with all treatment groups.
This increase, however, was significantly reversed in untreated animals (Figures $9 \& 10$ ). 


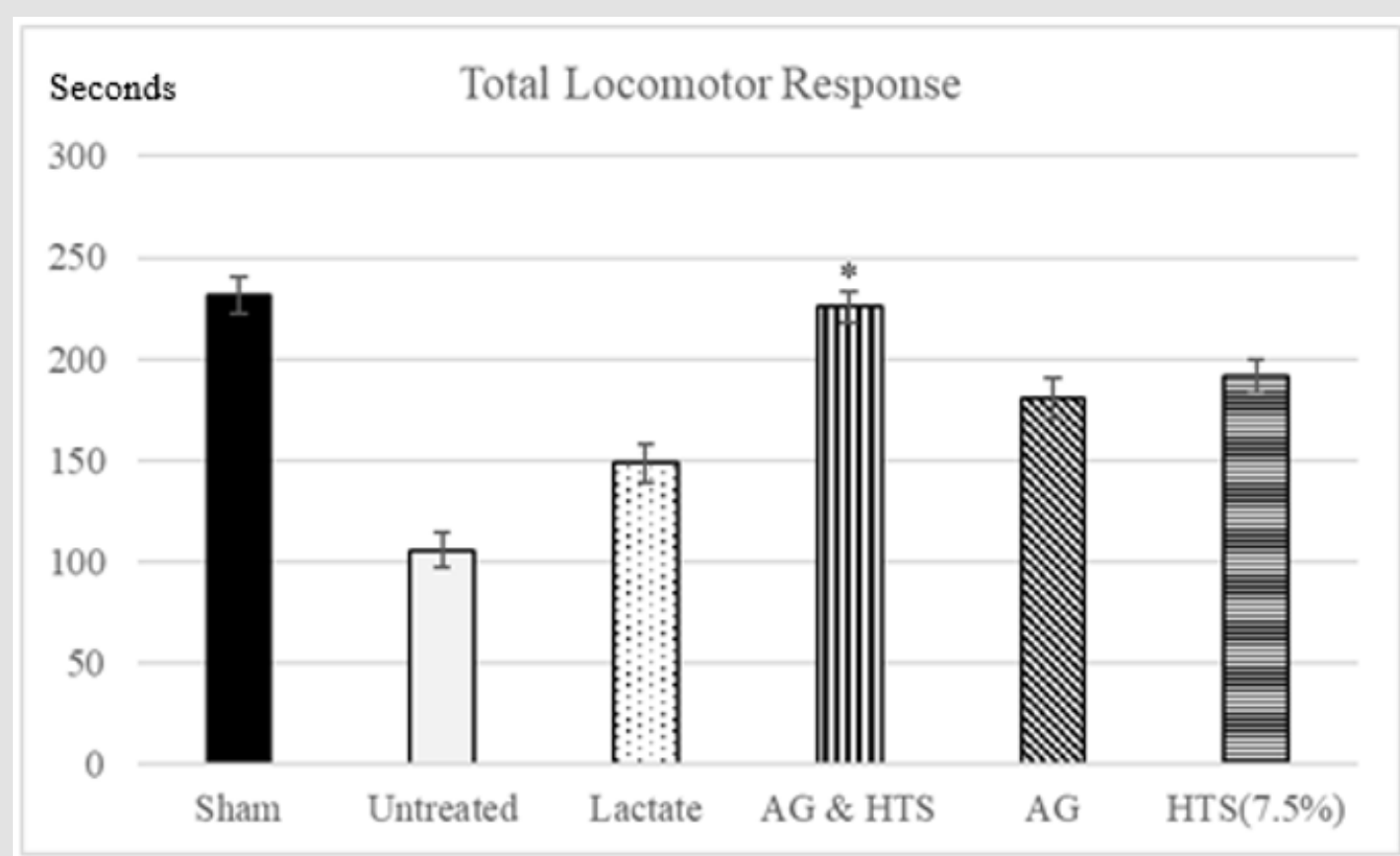

Figure 9: A significant increase in total was observed in the AG+HTS group when compared with untreated and all treatment groups. ( ${ }^{*} \mathrm{p}<0.05$ vs untreated and treated groups).

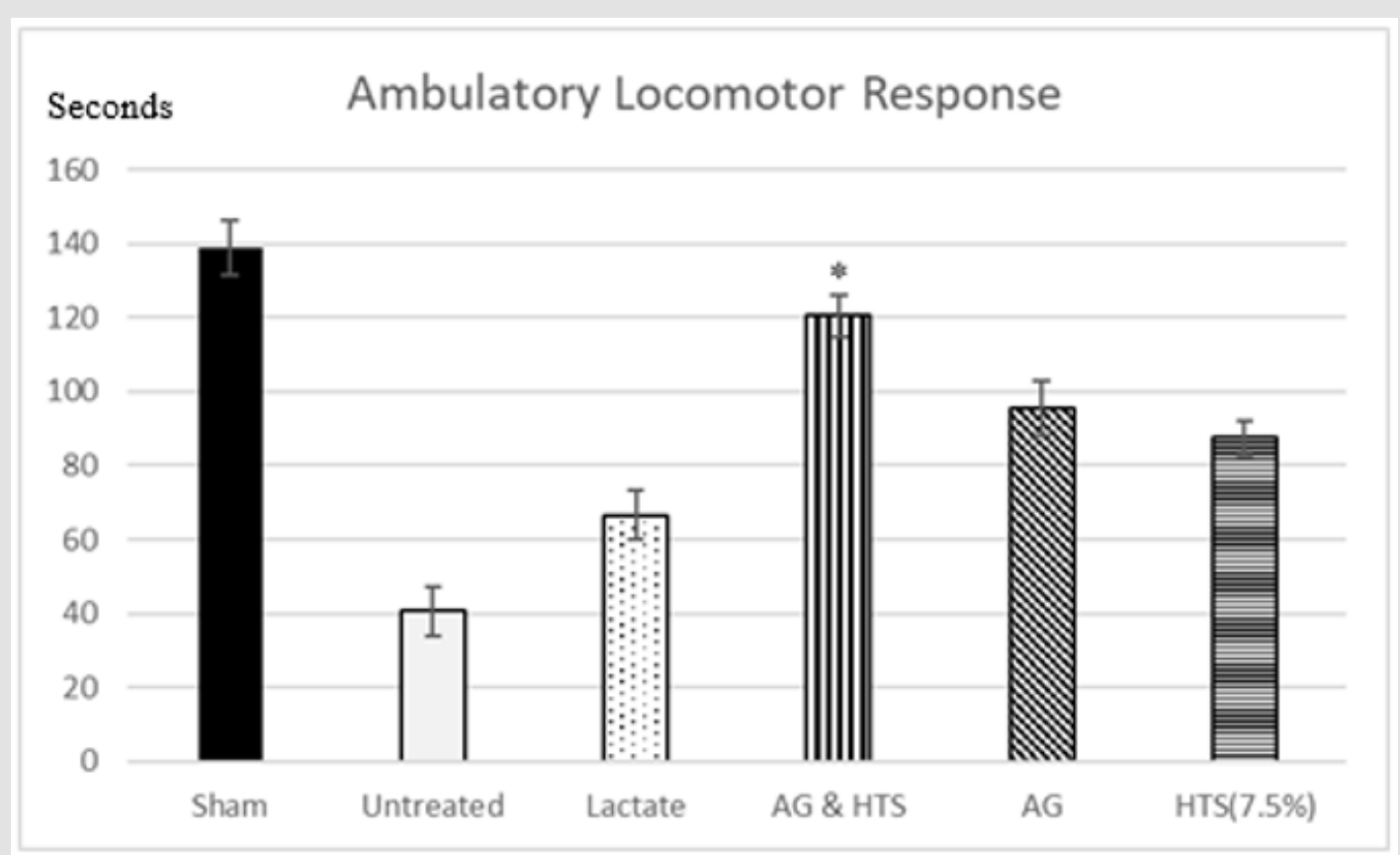

Figure 10: A significant increase ambulatory activity was observed in the AG+HTS group when compared with untreated and all treatment groups. ( ${ }^{*} \mathrm{p}<0.05$ vs untreated and treated groups).

Acoustic startle response (Figure 11): Group comparison of acoustic startle response showed no significant difference between sham-operated and AG+HTS rats. However, a marked increase in acoustic startle response was observed in untreated and all other treated rats. 


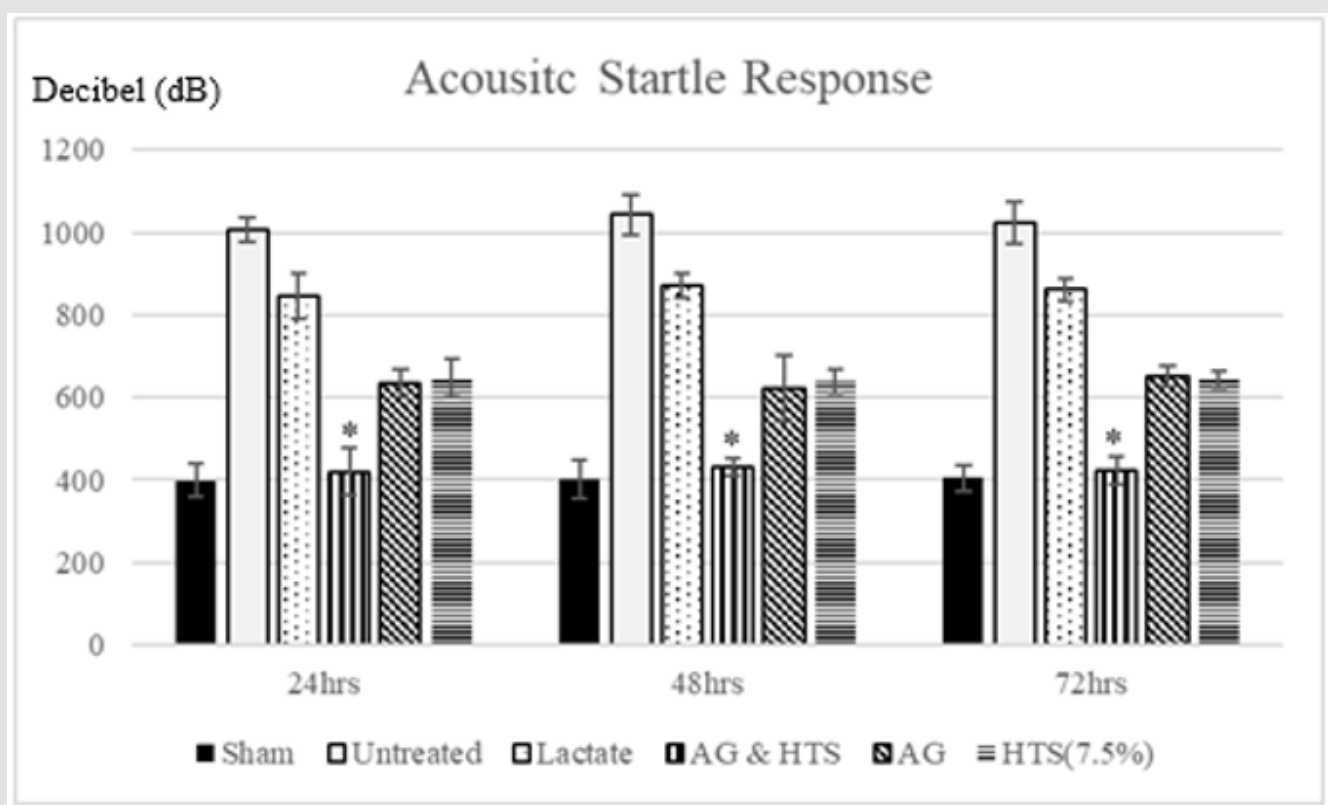

Figure 11: Group comparison of acoustic startle response showed no significant difference between sham-operated and AG+HTS rats. However, a marked increase in acoustic startle response was observed in untreated and all other treated rats. ( ${ }^{*} \mathrm{p}<0.05$ vs untreated and treated groups).

\section{Discussion}

Besides modulating the formation of various shock mediators by pharmacological agents, a major objective of fluid resuscitation in shock is the restoration of effective circulating intravascular volume. The ability to resuscitate with reduced fluid volume has been considered advantageous, in that it might reduce the potential consequences of third space fluid sequestration, such as the development of cerebral edema in the head-injured patient or pulmonary edema in the patient with contused lung [25]. Resuscitation with small volumes of HTS solutions has been shown to restore circulating volume and hemodynamics, in part through imbibement of intracellular fluid into the vascular space [26]. Further, some studies have reported improved cardiac contractility with HTS resuscitation regimens [27]. Both experimental and clinical trials have suggested a more favorable outcome following HTS resuscitation from hemorrhagic shock [26,27], although the mechanisms underlying this perceived benefit remain poorly elucidated. It is suggested that the hypertonic component draws water out of the intracellular space and acts to replenish the depleted extracellular space. The hypertonic state created by the solutions reduces edema in the endothelial cells of the microvasculature and enhances microcirculatory flow and, in the presence of experimental head injuries, recede the formation of cerebral edema [28-30]. The solution may also increase myocardial contractility [31].

Studies have shown that during the event of shock, NO synthase (iNOS) inhibitor show inhibition in NO overproduction that may contribute to the vascular impairment [32]. Animal studies have also suggested that nitric oxide (NO) overproduction may mediate vascular hypo reactivity and decompensation following hemorrhagic shock [33,34]. Neurotoxicity of NO overproduction in the brain has also been shown by several studies [35-37]. NO may play an important role in secondary neuronal damage after ischemia [38]. In a recent study, an iNOS inhibitor was shown to increase cardiac output in a model of hypovolemic haemorrhage [39]. Selective iNOS inhibitors have also been shown to increase survivability by improving hemodynamic indices and reducing organ injuries in hemorrhagic $[40,41]$ as well as endotoxic shock models $[42,43]$. AG is a more potent inhibitor of iNOS than NGsubstituted arginine analogues. It is approximately 30 times more selective to iNOS enzyme than enthothelial nitric oxide synthase (eNOS) enzymes [44,45]. In a recent study, AG had decreased serum nitrate/nitrite level and increased survival rate in an endotoxic and hemorrhagic shock model $[40,46]$. AG have been demonstrated in several experimental animal models of stroke and traumatic brain injury [22,38,47-49] and it was effective in reducing the ischemic lesion volume when administered before or after the injury $[22,38,49]$.

Our study showed that the AG+HTS was able to raise the MABP more than when shocked rats are treated with AG or HTS alone. This had translated into restoration of blood gas parameters (p02 and $\mathrm{pH}$ ) to almost physiological levels, reduced histological damages (reduction in GOT and nitrate/nitrite levels) and improved physical and behavioral performance. The use of AG and HTS could have brought about a synergistic effect that might have restored and effectively improved circulating intravascular volume. This in turn, might have prevented vascular hypo reactivity and decompensation by over production of NO by iNOS thus effectively attenuating hypotension in hemorrhagic shock. In conclusion, our results showed that administration of small volume of resuscitative fluid 
which contains aminoguanidine and hypertonic saline significantly improved the hemodynamic, histology and neurological parameters in anaesthetized rat hemorrhagic shock models.

\section{References}

1. Hooper N, Armstrong TJ (2020) Hemorrhagic Shock. In: StatPearls. Treasure Island (FL): StatPearls Publishing, NCBI.

2. Kowalski A, Brandis D (2020) Shock Resuscitation. In: StatPearls. Treasure Island (FL): StatPearls Publishing, NCBI.

3. Chipman AM, Jenne C, Wu F, Kozar RA (2020) Contemporary resuscitation of hemorrhagic shock: What will the future hold? [published online ahead of print, 2020 May 11]. Am J Surg S0002-9610(20): 30265-30268.

4. Alexi K, Ahmad M, Jacob G (2020) Hypertonic Fluids. StatPearls Publishing, NCBI.

5. Casey JD, Brown RM, Semler MW (2018) Resuscitation fluids. Current opinion in critical care 24(6): 512.

6. Ramesh GH, Uma JC, Farhath S (2019) Fluid resuscitation in trauma: what are the best strategies and fluids?. International Journal of Emergency Medicine 12(1): 1-6.

7. Powell K, Shah K, Hao C, Wu YC, A Jhon, et al. (2019) Neuromodulation as a new avenue for resuscitation in hemorrhagic shock. Bioelectron Med 5: 17 .

8. Dauer E, Goldberg A (2019) What's New in Trauma Resuscitation. Adv Surg 53: 221-233.

9. Jackson CD, Patel SB, Haltom MB (2019) Choice of Intravenous Crystalloid Fluid and Mortality in Critically Ill Adult Patients. South Med J 112(7): 401-405.

10. Mason AK, Malik A, Ginglen JG (2020) Hypertonic Fluids. In: StatPearls. Treasure Island (FL): StatPearls Publishing, NCBI.

11. Lambden S (2019) Bench to bedside review: therapeutic modulation of nitric oxide in sepsis-an update. Intensive Care Med Exp 7(1): 64.

12. Papi S, Ahmadizar F, Hasanvand A (2019) The role of nitric oxide in inflammation and oxidative stress. Immunopathologia Persa 5(1): e08-e08.

13. Gutierrez G, Reines H, Wulf Gutierre ME (2004) Clinical review: hemorrhagic shock. Critical care 8(5): 373.

14. Bedirli A, Sözüer EM, Muhtaroğlu S, Alper M (2000) The role of oxygen free radicals and nitric oxide in organ injury following hemorrhagic shock and reinfusion. Int J Surg Investig 2(4): 275-284.

15. Gulati A (2016) Vascular Endothelium and Hypovolemic Shock. Curr Vasc Pharmacol 14(2): 187-195.

16. Chen ZQ, Mou RT, Feng DX, Wang Z, Chen G (2017) The role of nitric oxide in stroke. Med Gas Res 7(3): 194-203.

17. Shirakawa H, Kaneko S (2018) Physiological and Pathophysiological Roles of Transient Receptor Potential Channels in Microglia-Related CNS Inflammatory Diseases. Biol Pharm Bull 41(8): 1152-1157.

18. Li JJ, Liu SJ, Liu XY, Ling EA (2018) Herbal Compounds with Special Reference to Gastrodin as Potential Therapeutic Agents for Microglia Mediated Neuroinflammation. Curr Med Chem 25(42): 5958-5974.

19. Wu MY, Yiang GT, Liao WT, Andy PT, Cheng YL, et al. (2018) Current Mechanistic Concepts in Ischemia and Reperfusion Injury. Cell Physiol Biochem 46(4): 1650-1667.

20. Alharbi BM, Tso MK, Macdonald RL (2016) Animal models of spontaneous intracerebral hemorrhage. Neurol Res 38(5): 448-455.

21. Hu X, Tao C, Gan Q, Zheng J, Li H, et al. (2016) Oxidative Stress in Intracerebral Hemorrhage: Sources, Mechanisms, and Therapeutic Targets. Oxid Med Cell Longev 2016: 3215391.
22. Guo ZN, Shao A, Tong LS, Sun W, Liu J, et al. (2016) The Role of Nitric Oxide and Sympathetic Control in Cerebral Autoregulation in the Setting of Subarachnoid Hemorrhage and Traumatic Brain Injury. Mol Neurobiol 53(6): 3606-3615.

23. Soliman MM (2014) Effects of aminoguanidine, a potent nitric oxide synthase inhibitor, on myocardial and organ structure in a rat model of hemorrhagic shock. Journal of emergencies, trauma, and shock 7(3): 190.

24. Pavlovic D, Pekic S, Stojanovic M, Popovic V (2019) Traumatic brain injury: neuropathological, neurocognitive, and neurobehavioral sequelae. Pituitary 22(3): 270-282.

25. Ozturk H, Yagmur Y, Tas A, Topcu S, Orak M (2007) Continuous infusion of small-volume fluid resuscitation in the treatment of combined uncontrolled hemorrhagic shock and head injury. J Coll Physicians Surg Pak 17(1): 19-22.

26. Wade CE, Grady JJ, Kramer GC (2003) Efficacy of hypertonic saline dextran fluid resuscitation for patients with hypotension from penetrating trauma. J Trauma 54(5 Suppl): S144-S148.

27. Ogino R (2002) Effects of hypertonic saline and dextran 70 on cardiac diastolic function after hemorrhagic shock. J Surg Res 107(1): 27-36.

28. Singh S, Davis D (2020) Ringer's Lactate. In: StatPearls. Treasure Island (FL): StatPearls Publishing, NCBI.

29. Xiao HP, Gu MN, Xiao JF, Xu X, Zhao ZL (2008) Nan Fang Yi Ke Da Xue Xue Bao 28(3): 385-388.

30. Nascimento P Jr, De Paiva Filho O, De Carvalho LR, Braz JR (2006) Early hemodynamic and renal effects of hemorrhagic shock resuscitation with lactated Ringer's solution, hydroxyethyl starch, and hypertonic saline with or without 6\% dextran-70. J Surg Res 136(1): 98-105.

31. Prough DS, Kramer GC, Uchida T, Stephenson RT, Hellmich HL, et al (2006) Effects of hypertonic arginine on cerebral blood flow and intracranial pressure after traumatic brain injury combined with hemorrhagic hypotension. Shock 26(3): 290-295.

32. Ng KC, Moochhala SM, Shirhan Md, Yap EL, Low SY (2003) Preservation of neurological functions by nitric oxide synthase inhibitors following hemorrhagic shock. Neuropharmacology 44: 244-252.

33. Md S, Moochhala SM, Siew Yang KL (2003) The role of inducible nitric oxide synthase inhibitor on the arteriolar hyporesponsiveness in hemorrhagic-shocked rats. Life Sci 73(14): 1825-1834.

34. Gulati A (2016) Vascular Endothelium and Hypovolemic Shock. Curr Vasc Pharmacol 14(2): 187-195.

35. Rodrigo J, Fernández AP, Serrano J, Peinado MA, Martínez A (2005) The role of free radicals in cerebral hypoxia and ischemia. Free Radic Biol Med 39(1): 26-50

36. Wu KW, Kou ZW, Mo JL, Deng XX, Sun FY (2016) Neurovascular coupling protects neurons against hypoxic injury via inhibition of potassium currents by generation of nitric oxide in direct neuron and endothelium cocultures. Neuroscience 334: 275-282.

37. Liaudet L, Soriano FG, Szabo C (2000) Biology of nitric oxide signaling Critical Care Medicine 28(4 (Suppl)): N37-N52.

38. Sun K, Fan J, Han J (2015) Ameliorating effects of traditional Chinese medicine preparation, Chinese materia medica and active compounds on ischemia/reperfusion-induced cerebral microcirculatory disturbances and neuron damage. Acta Pharm Sin B 5(1): 8-24.

39. Rajagopalan S, Kurz S, Münzel T, Tarpey M, BA Freeman, et al. (1996) Angiotensin II-mediated hypertension in the rat increases vascular superoxide production via membrane NADH/NADPH oxidase activation. Contribution to alterations of vasomotor tone. J Clin Invest 97(8): 1916-1923.

40. Atan MS, Moochhala SM, Ng KC, Low K, Teo AL, et al. (2004) Effects of aminoguanidine and L-arginine methyl ester resuscitation following 
induction of fluid-percussion injury and severe controlled hemorrhagic shock in the rat brain. J Neurosurg 101(1): 138-144.

41. Zingarelli B, Ischiropoulos H, Salzman AL, Szabo C (1997) Amelioration by mercaptoethylguanidine of the vascular and energetic failure in hemorrhagic shock in the anesthetized rat. European Journal of Pharmacology 338(1): 55-65.

42. Liaudet L, Feihl F, Rosselet A, Markert M, Hurni JM, et al. (1996) Beneficial effects of L-canavanine, a selective inhibitor of iNOS, during rodent endotoxaemia. Clinical Science 90(5): 369-377.

43. Umans JG, Samsel RW (1992) L-canavanine selectively augments contraction in aortas from endotoxemic rats. European Journal of Pharmacology 210(3): 343-346.

44. Corbett JA, Tilton RG, Chang K, Hassan KS, Ido Y, et al. (1992) Aminoguanidine, a novel of nitric oxide formation, prevents diabetic vascular dysfunction. Diabetes 41: 552-556.

45. Griffith MJD, Messent M, Mac Alister RJ, Evan TW (1993) Aminoguanidine selectively inhibits inducible nitric oxide synthase. British Journal Pharmacology 110: 963-968

ISSN: 2574-1241

DOI: $10.26717 / B J S T R .2020 .28 .004653$

Shabbir M Moochhala. Biomed J Sci \& Tech Res

CC (i) This work is licensed under Creative Commons Attribution 4.0 License

Submission Link: https://biomedres.us/submit-manuscript.php
46. Arkovitz MS, Wispe JR, Garcia VF, Szabo C (1996) Selective of the inducible isoform of nitric synthase prevents pulmonary transvascular flux during acute endotoxemia. Journal of Pediatric Surgery 31(8): 10091015.

47. Stoffel M, Rinecker M, Plesnila N, Eriskat J, Baethmann A (2000) Attenuation of secondary lesion growth in the brain after trauma by selective inhibition of the inducible NO-synthase. Acta Neurochirurgia Supplement 76: 357-358.

48. Wada K, Chatzipanteli K, Kraydieh S, Busto R, Dietrich WD (1998) Inducible nitric oxide synthase expression after traumatic brain injury and neuroprotection with aminoguanidine treatment in rats. Neurosurgery 43: 1427-1436.

49. Cash D, Beech JS, Rayne RC, Bath PM, Meldrum BS, et al. (2001) Neuroprotective effect of aminoguanidine on transient focal ischaemia in the rat brain. Brain Research 905: 91-103.

$\begin{array}{ll}\text { BIOMEDICAL } & \text { Assets of Publishing with us } \\ \text { RESEARCHES } & \text { - Global archiving of articles } \\ & \text { - Immediate, unrestricted online access } \\ \end{array}$

